MR. TUOMAS HÄMÄLÄ (Orcid ID : 0000-0001-8306-3397)

Article type : Original Article

\title{
Role of seed germination in adaptation and reproductive isolation in
}

\section{Arabidopsis lyrata}

Tuomas Hämälä ${ }^{1,2,3}$, Tiina M. Mattila ${ }^{1}$, Päivi H. Leinonen ${ }^{1,4}$, Helmi Kuittinen ${ }^{1}$ and Outi Savolainen $^{1,2}$

${ }^{1}$ Department of Ecology and Genetics, University of Oulu, FI-90014 Oulu, Finland

${ }^{2}$ Biocenter Oulu, University of Oulu, FI-90014 Oulu, Finland

${ }^{3}$ E-mail: tuomas.hamala@oulu.fi

${ }^{4}$ Current address: Natural Resources Institute Finland (Luke), Management and Production of Renewable Resources, FI-00790 Helsinki, Finland

Running head: Germination in adaptation and speciation

Keywords: Germination, speciation, adaptation, Bateson-Dobzhansky-Muller incompatibility, QTL mapping, transmission ratio distortion (TRD)

This article has been accepted for publication and undergone full peer review but has not been through the copyediting, typesetting, pagination and proofreading process, which may lead to differences between this version and the Version of Record. Please cite this article as doi: $10.1111 / \mathrm{mec} .14135$

This article is protected by copyright. All rights reserved. 


\begin{abstract}
Seed germination is an important developmental and life history stage. Yet, the evolutionary impact of germination has mainly been studied in the context of dormancy, or for its role in reproductive isolation between species. Here, we aim to examine multiple consequences of genetic divergence on germination traits between two Arabidopsis lyrata subspecies: ssp. petraea (Eurasia) and ssp. lyrata (North-America). Post-dormancy germination time, a potentially adaptive trait, showed differentiation between the populations, and quantitative trait loci (QTL) mapping revealed that the trait variation is mainly controlled by two antagonistic loci. These QTL areas contain several candidate genes with known function in post-dormancy germination in A. thaliana. The sequence variation of three genes was consistent with differential selection, and they also included fixed nonsynonymous substitutions with potential to account for the phenotypic differentiation. We further show that the divergence between the subspecies has led to a slight but significant reduction in hybrid germination proportions, indicating incipient reproductive isolation. Comparison of reciprocal $F_{1}$ and $F_{2}$ progenies suggest that Bateson-Dobzhansky-Muller-incompatibilities likely act through uniparentally inherited factors. Examination of genome-wide transmission ratio distortion further revealed that cytonuclear interactions cause substantial pregermination inviability in the hybrids. These results confirm that seed germination has adaptive potential beyond the dormancy stage and that hybrid seed inviability can be one of the first reproductive barriers to arise during divergence.
\end{abstract}

\title{
Introduction
}

The transition from seed to seedling is one of the first developmental phases experienced by a plant individual. Germination, consequently, has a direct influence on the success of the early life stages, but it also has cascading effects on survival and fecundity throughout the individuals' life (Donohue et al. 2010). As such, seed germination is often a prime subject for

This article is protected by copyright. All rights reserved. 
natural selection - a fact that can have a significant influence on local-adaptation (Kim \& Donohue 2013; Postma \& Ågren 2016) and reproductive isolation (Tiffin et al. 2001; Lowry et al. 2008).

Timing of seed germination has profound ecological consequences, as it defines the growing season and in part the environment for the vulnerable seedling stage. Indeed, germination timing often varies between populations (Debieu et al. 2013) and environments (Montesinos-Navarro et al. 2012), frequently contributing to adaptive divergence (Donohue et al. 2010; Kim \& Donohue 2013; Postma \& Ågren 2016). The main factor controlling germination timing is seed dormancy, a physiological state during which viable seed does not germinate even if other conditions were to allow it (Finch-Savage \& Leubner-Metzger 2006). Through extensive studies in Arabidopsis thaliana, and other species, we know that warm and dry environments are generally associated with higher levels of dormancy and shorter growth periods with lower levels of dormancy (Donohue et al. 2010; Wagmann et al. 2012; Debieu et al. 2013). At the genetic level, dormancy in A. thaliana seems to be primarily controlled by DELAY OF GERMINATION1 (DOG1), a gene that has been found in several quantitative trait loci (QTL) mapping experiments (Alonso-Blanco et al. 2003; Bentsink et al. 2010; Postma \& Ågren 2015, 2016), and studied in detail (Bentsink et al. 2006; Kronholm et al. 2012; Kerdaffrec et al. 2016). Divergence in dormancy release is therefore important in certain contrasting environments, but even without dormancy differences, variation in postdormancy germination traits may affect fitness by influencing growth and survival of seedlings in their first growing season. However, little is known about phenotypic variation or genetic basis of these traits.

Besides its potential in adaptation, germination can have an evolutionary impact when reproductive isolation results in reduced hybrid seed viability. Hybrid seed failure is a common result of interploidy crosses (Haig \& Westoby 1991; Ramsey \& Schemske 1998),

This article is protected by copyright. All rights reserved. 
but it has also been found to be a significant reproductive barrier between diploid species in Mimulus (Garner et al. 2016; Oneal et al. 2016), Capsella (Rebernig et al. 2015) and Solanum (Florez-Rueda et al. 2016). Recent studies have also provided some first evidence that hybrid seed inviability can form between different populations of the same species (Barnard-Kubow et al. 2016). Seed failure in flowering plants is commonly attributed to faults in endosperm development (Brink \& Cooper 1947; Haig \& Westoby 1991). This nutritive tissue is born out of double fertilization: two male nuclei reach the female gametophyte, where one fertilizes the egg to produce a zygote and the other fuses with two central cell nuclei to form a triploid endosperm. Deviations from the 2:1 maternal to paternal genome ratios are known to disrupt embryo development and to cause hybrid seed mortality in crosses between different ploidy levels (Ramsey \& Schemske 1998). In diploid crosses, hybrid embryo arrest can be caused by negative interactions between epistatic alleles of different loci, commonly called Bateson-Dobzhansky-Muller-incompatibilities (BDMI) (Bateson 1909; Dobzhansky 1936; Muller 1939), in the endosperm or in the zygote (Oneal et al. 2016). The negatively interacting alleles are often part of the nuclear genome, but a growing number of investigations have shown that BDMIs can form between the nuclear genome and the organellar genomes of the mitochondria or chloroplast (Tiffin et al. 2001; Levin 2003; Burton et al. 2013). These incompatibilities are thought to be especially important during early stages of the speciation process, because the faster structural evolution and higher genetic drift of organelle genomes, as well as conflicts associated with coevolution between organelle and nuclear genes, may enable them to form before nuclear incompatibilities (Levin 2003; Burton et al. 2013).

In the present study, we examined phenotypic differentiation in germination traits and signs of reproductive isolation in hybrid seed viability between two Arabidopsis lyrata subspecies. These subspecies occur in allopatry: ssp. petraea is found in Eurasia and

This article is protected by copyright. All rights reserved. 
ssp. lyrata in North-America (Jalas \& Suominen 1994). The lineages split about 200,000 years ago (Pyhäjärvi et al. 2012) and now the genetic differentiation between the subspecies is considerable $\left(F_{\mathrm{ST}}>0.6\right)$ (Muller et al. 2008; Pyhäjärvi et al. 2012; this study). Both subspecies contain several isolated populations that are locally adapted (Leinonen et al. 2009, 2011; Vergeer \& Kunin 2013) and phenotypically diverged in several reproductive and morphological traits (Kärkkäinen et al. 2004; Riihimäki \& Savolainen 2004; Quilot-Turion et al. 2013; Remington et al. 2015). Earlier studies have shown that progenies between the subspecies, as well as between populations within subspecies, have many loci with distorted allele or genotype frequencies (called transmission ratio distortions [TRD]) (Kuittinen et al. 2004; Leppälä \& Savolainen 2011; Aalto et al. 2013; Leppälä et al. 2013). The number of distorted loci within populations is low (Leppälä et al. 2008), suggesting that TRD in interpopulation crosses is likely caused by BDMIs between alleles from diverged populations. Despite the divergence and TRD, crosses between the subspecies produce viable progeny that have shown hybrid vigor and surpassed the pure-population individuals in postgermination fitness (Leinonen et al. 2011). However, progeny from the same crosses have also shown evidence of severe cytoplasmic male sterility (Leppälä \& Savolainen 2011; Aalto et al. 2013), raising questions about the extent of reproductive isolation between these subspecies and whether other traits than fertility are affected by the divergence.

To examine the evolutionary significance of germination in A. lyrata comprehensively, we addressed questions concerning its role in adaptation and reproductive isolation: Do we see evidence of phenotypic differentiation in germination traits between the A. lyrata subspecies? What kind of genetic architecture underlies these traits? Do hybrids between the subspecies have delayed germination times or lower germination proportions compared to parental populations? What genetic mechanisms cause the lowered hybrid seed viability? And, are the genetic incompatibility alleles nuclear or do we see evidence of

This article is protected by copyright. All rights reserved. 
cytonuclear interactions or other asymmetry causing factors? We conducted a reciprocal crossing experiment between populations from two highly different environments: Karhumäki, Russia (Lat $62^{\circ} \mathrm{N}$ ) and Mayodan, North Carolina (Lat $36^{\circ} \mathrm{N}$ ). The two habitats differ in temperatures, rainfalls and annual variation in day lengths, possibly leading to different germination responses. Seed traits were examined by quantifying germination rates in pure-populations as well as in their $F_{1}$ and $F_{2}$ hybrid progeny. QTL for germination time and TRD loci potentially underlying the genomic incompatibilities were mapped in two independent $\mathrm{F}_{2}$ families with dense marker maps acquired using double digest RAD (ddRAD) sequencing (Peterson et al. 2012). We then used whole-genome resequencing data from purepopulation individuals to analyze nucleotide variation in any candidate genes found within the QTL areas.

\section{Materials and methods}

\section{Populations and crosses}

Two populations of Arabidopsis lyrata were used in this study: one from Karhumäki, Russia $\left(62^{\circ} 55^{\prime} \mathrm{N}, 34^{\circ} 25 \mathrm{E}\right)$, representing the European subspecies ssp. petraea and one from Mayodan, North Carolina $\left(36^{\circ} 25^{\prime} \mathrm{N}, 7^{\circ} 58^{\prime} \mathrm{W}\right)$, representing the North American subspecies ssp. lyrata. The two locations are close to the northern and southern range limits of A. lyrata (Jalas \& Suominen 1994), and consequently, the growing season length (monthly mean temperature $>5^{\circ} \mathrm{C}$ ) is substantially longer in Mayodan (from March to November) than in Karhumäki (from April to September). The mean annual temperature and annual precipitation are also highly different: $1.9^{\circ} \mathrm{C}$ and $559 \mathrm{~mm}$ for Karhumäki, and $14.4^{\circ} \mathrm{C}$ and $1129 \mathrm{~mm}$ for Mayodan, respectively [data from WorldClim (Hijmans et al. 2005)]. Despite the higher annual rainfall, the aridity index (AI; mean annual precipitation / potential evapotranspiration) indicates that Mayodan $(\mathrm{AI}=0.91)$ is still less humid than Karhumäki $(\mathrm{AI}=1.12)$ (Trabucco \& Zomer 2009).

This article is protected by copyright. All rights reserved. 
Several independent individuals from Karhumäki (abbreviated as Kar from here on) and Mayodan (abbreviated as Ma from here on) were crossed to produce new within- and between-population seed families. Individuals from Kar were pollinated with Ma pollen to generate $\mathrm{F}_{1}$ progeny with Kar cytoplasm (abbreviated as $\mathrm{F}_{1}$-KarMa) and Ma individuals were pollinated with Kar pollen to produce $F_{1}$ progeny with Ma cytoplasm (abbreviated as $F_{1-}$ MaKar). Independent $F_{1}$ plants with different cytoplasms were then chosen and crossed reciprocally to generate $F_{2}$ families with both Kar and Ma cytoplasms (abbreviated as $\mathrm{F}_{2-}$ KarMa and $\mathrm{F}_{2}$-MaKar, respectively). This crossing design was used to avoid inbreeding depression caused by the obligate outcrossing nature of these populations of A. lyrata and to produce crossing progeny with different cytoplasms. The following number of full-sib families were generated for the experiment: "Kar" $n=3$, "Ma" $n=4$, " $F_{1}-K_{a r M a " ~} n=6$, " $F_{1}$ -

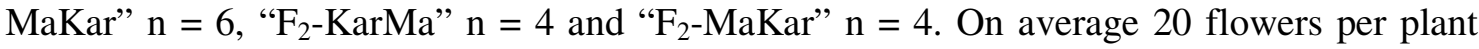
were pollinated by hand in pollinator-free conditions. Seeds matured in growth chamber conditions with $+20^{\circ} \mathrm{C}$ temperature and $16 \mathrm{~h}$ light $/ 8 \mathrm{~h}$ dark -cycle. Ripe seeds were collected in paper bags, counted and stored in growth cabinet (Sanyo MLR 350H), in stable conditions $\left(+23^{\circ} \mathrm{C}\right.$ temperature, $30 \%$ relative humidity and continuous dark $)$, until the start of the germination experiment.

\section{Germination experiment}

Germination differences between parental populations and $F_{1}$ and $F_{2}$ hybrid progeny were assessed by planting seeds from each group and monitoring their germination in controlled conditions. To evaluate potential dormancy difference between Kar and Ma, and between hybrids and pure-populations, we used two after-ripening times and two cold stratification treatments before the experiment. Both treatments are known to decrease dormancy in $A$. thaliana (Baskin \& Baskin 1998; Penfield \& Springthorpe 2012; Debieu et al. 2013), and by modifying treatment times, we can obtain an estimate of dormancy responses in each group.

This article is protected by copyright. All rights reserved. 
The experiment was performed in two parts: the first part included seeds that were kept in after-ripening (dry storage in stable conditions) for eight weeks and the second part included seeds that were kept in after-ripening for 19 weeks. Seeds that received the two cold stratification treatments (four and eight days) were included in both parts. 100 seeds from each seed family ( 25 per treatment), except for one $F_{1}$-KarMa family in which only 63 seeds were available, (2,663 seeds in total) were randomized into Petri plates filled with 5\% agar and stratified in $+4^{\circ} \mathrm{C}$. After stratification, plates were moved into a growth cabinet (Sanyo MLR $350 \mathrm{H}$ ), with $+20^{\circ} \mathrm{C}$ temperature, $8 \mathrm{~h}$ light $/ 16 \mathrm{~h}$ dark -cycle, and $80 \%$ relative humidity. Plates were randomized within the cabinet and the order was changed daily. Germination was monitored daily for 28 days. A seed was scored as germinated when a visible root penetrated the seed coat.

\section{Statistical analysis}

Germination was quantified using two traits: germination time, measured as days from the start of the experiment (when plates were moved into the growth cabinet) until root emergence, and germination probability, measured as proportion of germinated seeds. Traits were analyzed with general and generalized linear mixed models in package lme4 v1.1-12 (Bates et al. 2016) in $\mathrm{R}$ v3.2.3 (R Core Team 2015) following Leinonen et al. (2011).

Germination times were modeled as normally distributed (data was log-transformed to improve homoscedasticity) with identity link function, and for germination proportions we fitted a binomial distribution with logit link function. Population (Kar, Ma, $\mathrm{F}_{1}-\mathrm{KarMa}, \mathrm{F}_{1}$ MaKar, $\mathrm{F}_{2}$-KarMa and $\mathrm{F}_{2}$-MaKar) as well as after-ripening and stratification time treatments were modeled as fixed effects and plate and family as random effects (representing environmental variation and genetic variation within populations, respectively). Significance of the different factors and pairwise-differences between populations were evaluated using likelihood-ratio tests by comparing the fit of a full model to a reduced model with the

This article is protected by copyright. All rights reserved. 
variable of interest removed or a pair of populations combined as one category. Stratification had a significant effect on germination time, so it was included as a cofactor in the pairwise comparison models.

\section{Library preparation and sequencing}

After the germination experiment, $F_{2}$ seeds from two families (both consisting of two reciprocals) were moved to pots and grown until big enough for leaf collections. These reciprocals included the following number of individuals: "KarMa1" $\mathrm{n}=87$, "MaKar1" $\mathrm{n}=$ 85, "KarMa2" $\mathrm{n}=93$ and "MaKar2" $\mathrm{n}=89$. Genomic DNA was extracted from dried leaves using DNeasy 96 Plant kits (Qiagen) and double-digested using restriction enzymes $M$ feI and NlaIII (New England Biolabs) according to manufacturer's instructions. Library preparation for double-digest restriction site-associated DNA (ddRAD) sequencing then followed the protocol described in Peterson et al. (2012). Sequencing was done with Illumina HiSeq2500 in Institute of Molecular Medicine Finland (FIMM) Technology Centre, University of Helsinki with 100 bp paired-end reads.

The parents of our crossing progeny were not included in the RAD sequencing libraries and we used whole-genome resequencing data from independent $\operatorname{Kar}(\mathrm{n}=4)$ and Ma $(n=6)$ individuals to assign population ancestry for our markers and to study sequence variation in candidate genes found within the QTL areas. Total genomic DNA was extracted from fresh leaves using NucleoSpin Plant II (Macherey-Nagel) kit and sent to the Institute of Molecular Medicine Finland (FIMM) Technology Centre, University of Helsinki for library preparation and sequencing.

This article is protected by copyright. All rights reserved. 


\section{Variant calling}

Raw data from RAD and whole-genome sequencing were first filtered to remove low-quality reads and Illumina adapters using Trimmomatic v0.35 (Bolger et al. 2014). Reads were then aligned to A. lyrata v1.0.24 reference genome (Hu et al. 2011) using BWA-MEM v0.7.8 (Li \& Durbin 2009; Li 2013). Picard tools v2.1.1 (http://broadinstitute.github.io/picard/) was used to remove duplicated reads, sort the data and to add read groups. Variant calling was done with FreeBayes v1.0.2 (Garrison \& Marth 2012), using only reads that had mapping quality over 20. A VCF-file was then filtered with VCFtools v0.1.13 (Danecek et al. 2011): SNPs and indels (only SNPs were used for the QTL and TRD analysis) with more than two alleles were removed, sites and genotypes were required to have a quality of over 20 and read depths between five and 100. Only markers that had both alleles unambiguously assigned to one of the populations (i.e. one allele is only found in the Kar whole-genome set and the other allele is only found in the Ma whole-genome set) were kept. We also excluded variants from reads that aligned to A. thaliana organelle genomes to avoid problems caused by possible errors in the A. lyrata reference genome assembly.

\section{QTL mapping}

Linkage maps for the two families were constructed in JoinMap 4.1 (Van Ooijen 2011), with 10.0 logarithm of odds (LOD) threshold, Kosambi's mapping function and regression algorithm with standard settings. Genome-wide analysis for germination time QTL was then performed in R/qtl (Broman et al. 2003). The two families were analyzed separately with Haley-Knott regression (Haley \& Knott 1992), using the automated stepwise search algorithm for model selection (Manichaikul et al. 2009). 1000 permutations were used to determine the genome-wide significance level at $\alpha=0.05$ for the QTL. Cytoplasm was taken into account by including it as an additive covariate in the models and possible cytonuclear

This article is protected by copyright. All rights reserved. 
interactions were analyzed by including cytoplasm also as an interactive covariate and comparing the peak LOD scores between the additive and additive + interactive models. A significance threshold for the interaction model was acquired by subtracting the values for peak LOD scores between the additive and additive + interactive models. In the case of cytonuclear interactions, we tested if the QTL had significant effect only in one reciprocal. Lastly, the proportion of phenotypic variance explained by each QTL and 95\% Bayesian credible intervals for their locations were estimated.

\section{Analysis of candidate genes}

We first searched the Ensembl (http://plants.ensembl.org/index.html) database for genes with known function in seed germination that localized within the $95 \%$ confidence intervals of the QTL. The Kar and Ma whole-genome datasets were then used to calculate $F_{\text {ST }}$ estimates for these genes using the Weir \& Cockerham (1984) method in VCFtools (Danecek et al. 2011). The estimates were compared against genome-wide $F_{\mathrm{ST}}$ distribution to evaluate whether these genes have higher genetic divergence than expected under neutrality (exceeded or were close to the two-tailed 5\% empirical threshold). For genes with high $F_{\mathrm{ST}}$ estimates, we then looked for nucleotide variants fixed between the populations. Based on information from Ensembl, we defined if the variants were located in coding areas, whether they changed the amino-acid composition of the peptide, and, in the case of nonsynonymous point-mutations, whether the change was to an amino-acid with similar (conservative mutation) or dissimilar (nonconservative mutation) biochemical properties. $F_{\mathrm{ST}}$ for the candidates were calculated using all variants found within the gene areas (between 20 and 71 SNPs or indels), and the genomewide estimates were calculated in 4-kb sliding windows (the average length of the candidate genes) for $4.5 \times 10^{6}$ variants.

This article is protected by copyright. All rights reserved. 


\section{Analysis of transmission ratio distortion}

Because TRD can be caused by epistatic interactions between two or more loci, we analyzed the distortion for nuclear one- and two-locus genotypes separately. Analysis was also done separately for the two reciprocals to distinguish possible interactions between cytoplasmic and nuclear genomes. For two-locus tests, the expected genotype frequencies were calculated by cross tabulating all marker pairs so that the single-locus genotype frequencies were taken into account. The significance of the deviation between the observed genotype ratios and those expected under Mendelian segregation was tested with standard $\chi^{2}$ tests for single-locus $($ d.f. $=2)$ and two-locus (d.f. $=4)$ genotypes. We used the conventional $P$-value threshold of 0.05 and a more stringent threshold of 0.001 . With high number of tests this undoubtedly leads to some false-positives, but with linked markers, standard corrections for multiple testing would lead to exceedingly high levels of false-negatives.

We used SNPs called from the next generation sequence alignments to analyze TRD, and while this allows for dense marker sets and accurate localization of the markers, it may create its own type of bias. The specimen for the A. lyrata reference genome is from the North American lyrata subspecies and is therefore more similar to Ma than to Kar individuals, possibly biasing the genotype ratios through preferential alignment of the reads. Around 85 $95 \%$ of the filtered reads in each progeny aligned successfully to the reference genome, a value similar to pure-population individuals, and we only used SNPs from areas that aligned in both parental populations. We also examined alignments in all heterozygote sites in the $\mathrm{F}_{2}$

data set and found only a slight difference between Kar and Ma allele read depths (on average: $52 \% \mathrm{Ma}$ and $48 \% \mathrm{Kar}$ ). Therefore, the effects of preferential alignment on our results should be negligible.

This article is protected by copyright. All rights reserved. 


\section{Results}

\section{Effects of after-ripening and stratification treatments on seed dormancy}

Stratification had a small but significant main effect on germination time $\left(\chi^{2}=5.65, P=0.018\right)$. After the four-day treatment, average germination time for the pure-populations and hybrids combined was 6.3 days and after the eight-day treatment, it was 6.8 days. Therefore, longer stratification led to slightly slower germination, possibly indicating incipient effects of secondary dormancy (Penfield \& Springthorpe 2012; Debieu et al. 2013). The treatment $\times$ population comparison, however, was not significant $\left(\chi^{2}=3.66, P=0.599\right)$. After-ripening had no effect on germination time, and neither after-ripening nor stratification influenced germination proportions (Table S1, Supporting information).

\section{Germination time}

Germination time, here defined as days from the start of the experiment (when plates were moved into a growth cabinet) until germination, showed significant differences between the pure-populations and between reciprocals in the first hybrid generation (Fig 1A and Table S2, Supporting information). There were no systematic differences between hybrids and purepopulations in germination time. Kar $($ mean $=5.3, \mathrm{SE}=0.19$, variance $=11.0)$ and $\mathrm{F}_{1}-\mathrm{KarMa}$ $($ mean $=5.1, \mathrm{SE}=0.13$, variance $=8.8)$ groups germinated more rapidly than Ma $($ mean $=7.4$, $\mathrm{SE}=0.15$, variance $=9.2)$ and $\mathrm{F}_{1}-\mathrm{MaKar}($ mean $=7.1, \mathrm{SE}=0.16$, variance $=14.6)$ groups, whereas $\mathrm{F}_{2}-\mathrm{KarMa}($ mean $=7.5, \mathrm{SE}=0.28$, variance $=27.8)$ and $\mathrm{F}_{2}$-MaKar $($ mean $=7.1, \mathrm{SE}$ $=0.26$, variance $=23.9)$ had similar germination times .

This article is protected by copyright. All rights reserved. 


\section{Germination proportions}

The two parental populations had the highest germination proportions: $0.993(\mathrm{SE}=0.005)$ for Kar and $0.967(\mathrm{SE}=0.009)$ for $\mathrm{Ma} . \mathrm{F}_{1}$ seed families had a moderate, but statistically significant, reduction in germination proportions when compared to pure populations (mean $=0.935, \mathrm{SE}=0.007)$ and a clear difference when compared to $\mathrm{F}_{2}$ hybrids, which had even lower germination proportions $($ mean $=0.872, \mathrm{SE}=0.012$ ). There were also distinct differences between the reciprocals of the $F_{1}$ crosses (Fig. 1B): $F_{1}$ seeds with Kar cytoplasm $\left(\mathrm{F}_{1}\right.$-KarMa) had germination proportion of $0.908(\mathrm{SE}=0.012)$, a value significantly lower than that of $F_{1}$ seeds with Ma cytoplasm $\left(F_{1}-\mathrm{MaKar}\right): 0.960(\mathrm{SE}=0.008)$. In the $\mathrm{F}_{2}$ generation, the reciprocals did not differ significantly from each other: $\mathrm{F}_{2}$-KarMa, 0.887 (SE $=0.016)$ and $\mathrm{F}_{2}-\mathrm{MaKar}, 0.857(\mathrm{SE}=0.017)$ (Fig. 1B and Table $\mathrm{S} 2$, Supporting information). Therefore, hybrids had lower germination proportions than pure-populations and $\mathrm{F}_{2}$ seeds lower than $F_{1}$ seeds, and germination reduction showed significant asymmetry in the first hybrid generation, but not in the second.

\section{Genome-wide scan for germination time QTL and analysis of candidate genes}

We used 1520 (family 1; $\mathrm{n}=172$ ) and 1794 (family 2; $\mathrm{n}=182$ ) high quality SNPs for the linkage map construction and QTL analysis. Markers were approximately evenly distributed throughout the chromosomes, leading to comprehensive coverage of the genome. Some chromosomes, however, had gaps in them (most notable in the middle of chromosome 6 [Fig. $2]$ ). This could be the result of TRD clustering (see next section), and if the missing genotypes in those areas were to be associated with the phenotype, it might also hamper the QTL discovery. Nevertheless, our QTL analysis discovered five regions at $\alpha=0.05$ linked to trait variation (Table 1 and Fig. 2). At two of these (Q3 and Q4), alleles from each population had large but opposite effects on phenotypic variation, and were shared between both families

This article is protected by copyright. All rights reserved. 
(Fig 3). Three QTL were discovered only in family 2, with Q5 and Q7 having cytonuclear interactions with significant effect only in Kar background.

Ten candidate genes were found within the $95 \%$ confidence intervals of the two QTL that occurred in both families (Table S3, Supporting information). The $F_{\text {ST }}$ comparisons showed that the genetic divergence estimates of three genes fell well beyond the mean $\left(F_{\mathrm{ST}}=\right.$ 0.646) values of the genome-wide distribution (Fig. S1 and Table S3, Supporting information): SPINDLY (SPY), $F_{\mathrm{ST}}=0.948$, corresponding to Q3 and FAR-RED ELONGATED HYPOCOTYL1 (FHY1), $F_{\mathrm{ST}}=0.937$ and MORE AXILLARY GROWTH2 $(M A X 2), F_{\mathrm{ST}}=0.987$, corresponding to $\mathrm{Q} 4$. Two fixed nonsynonymous point-mutations were discovered in $S P Y$ (one non-conservative and one nonsense mutation that shortens the peptide by 23 amino-acids in Kar individuals), one in FHY1 (non-conservative) and nine in MAX2 (seven conservative and two non-conservative) (Table S4, Supporting information).

\section{Transmission ratio distortion}

Our SNP sets for the TRD analysis included the following number of markers: KarMa1, 2389; MaKar1, 1702; KarMa2, 2364 and MaKar2, 2690. Under Mendelian segregation in the $F_{1}$ meiosis, and no zygotic selection, we expect these biallelic SNPs to have three genotypes (Kar-homozygote, KarMa-heterozygote, Ma-homozygote) in ratios 1:2:1. However, both families and both reciprocals had markers with distorted genotype ratios. The two $F_{2}$ reciprocals with Kar cytoplasm had the lowest percentages of distorted markers: $27 \%$ and $36 \%$ of markers deviated from the expected ratios at $\alpha=0.05$ and $12 \%$ and $17 \%$ at $\alpha=0.001$.

Levels of distortion were markedly higher for the two $\mathrm{F}_{2}$ reciprocals with Ma cytoplasm: $48 \%$ and $51 \%$ of the markers were distorted at $\alpha=0.05$ and $25 \%$ and $30 \%$ showed significant distortion at $\alpha=0.001$.

This article is protected by copyright. All rights reserved. 
To identify the locations of the distorted areas, we used information from the sequence alignments to plot each marker along the A. lyrata genome (Fig. 4). Again, the two reciprocals showed large differences in the locations of the TRD. For reciprocals with Kar cytoplasm, distorted markers were approximately evenly distributed along the genome, except for an area at the end of chromosome 5 and partly in chromosome 6 . The reciprocals with Ma cytoplasm, in contrast, showed clear clustering of significantly distorted markers in specific areas in chromosomes 2, 4 and, to a lesser extent, in chromosomes 5 and 6 . Areas of strong distortion in chromosomes 2, 4 and 6 in reciprocals with Ma cytoplasm were shared between both seed families, whereas other TRD areas in both reciprocals were due to distortion in only one of the families (Fig. S2, Supporting information). Analysis of the genotype frequencies showed an apparent deficiency of Kar homozygotes, especially in the most heavily distorted areas in chromosomes 2, 4 and 6 (Fig. 5 and Supporting information figures S3-S6).

Transmission ratio distortion was also analyzed for two-locus genotypes. The most significantly distorted areas $(P<0.001)$ were in reciprocals with Ma cytoplasm between chromosomes 2 and 4 (in family 1) and between chromosomes 4 and 5 (in family 2 and to a lesser extent in family 1). Overall, the number of loci involved in significant two-locus TRD was fairly small and they did not show much clustering (Fig. S7, Supporting information).

\section{Discussion}

We studied the genetic basis of within species variation in seed germination, an important life history and developmental trait, from two perspectives. Germination time revealed potentially adaptive differentiation and germination proportions evidence of reproductive isolation between A. lyrata subspecies.

This article is protected by copyright. All rights reserved. 


\section{Phenotypic differentiation in germination time}

There is ample evidence that timing of germination can influence fitness of an individual (Donohue et al. 2010; Kim \& Donohue 2013; Postma \& Ågren 2016). This is important so that seedlings can emerge during a suitable season, and in most cases it is facilitated by seed dormancy (Donohue et al. 2010). In earlier A. thaliana studies, populations have shown large dormancy differences between ten and 20 weeks of after-ripening, with southern populations generally exhibiting higher levels of dormancy than northern ones (Kronholm et al. 2012; Debieu et al. 2013; Postma \& Ågren 2015). Based on the latitudes and characteristics of their growing environments, we also expected to see higher levels of dormancy in the Ma population (seeds might be dispersed in the spring and stay dormant until early fall), while Kar seeds are likely to mature later in the season and germinate at a faster rate. However, the eight and 19 weeks after-ripening treatments had no effect on germination traits, and almost all pure-population seeds germinated during the experiment, indicating that eight weeks of after ripening was already enough to break dormancy in both populations. The maternal environments are, however, known to influence dormancy levels (Postma \& Ågren 2015; Burghardt et al. 2016), and the temperate growth chamber conditions during seed maturation may have reduced dormancy in one or in both of the populations (Burghardt et al. 2016). Nevertheless, the low levels of dormancy under these conditions suggest that Kar and Ma individuals can germinate without extended dormancy periods and overwinter as rosettes; the same strategy expressed by Central-European A. lyrata populations (Clauss \& Koch 2006). Therefore, post-dormancy germination time could have a crucial influence on growth and survival of seedlings in their first growing season before winter.

Previous studies in A. lyrata have shown that populations between and within subspecies differ in their flowering phenology. Northern populations generally benefit from a period of cold temperatures as rosettes (vernalization) to induce flowering (Riihimäki \&

This article is protected by copyright. All rights reserved. 
Savolainen 2004; Kemi et al. 2013), after which they open flowers faster than southern populations (Leinonen et al. 2011; Quilot-Turion et al. 2013). This faster vegetative to reproductive transition is often beneficial when the growing season in short, so the variation in post-dormancy germination may also be selected for faster seed to seedling transition in the northern Kar population. Alternatively, the slower germination of Ma seeds may be caused by thicker and less permeable seed coat (Baskin \& Baskin 1998), and it could be linked to adaptation to warmer and drier growing environments.

\section{Genetic basis of germination time differences}

The genome-wide scan for germination time QTL discovered five regions underlying the trait variation, of which two (Q3 and Q4) had large effects and were shared between both families. In fact, the difference in mean germination times between homozygotes in both QTL exceeded the difference between population averages by a clear margin (Fig. 1A and Fig. 3). This pattern is likely explained by the antagonistic effects of population specific alleles at Q3 and Q4. If both loci are fixed in Kar and Ma, they drive the phenotypic averages towards each other, but hybrids can have any combination of alleles in the two loci, and may exhibit extreme phenotypes relative to parents (Rieseberg et al. 2003). The $\mathrm{F}_{2}$ generation also had 2.5 times higher trait variance than parental populations, which further supports this conclusion. Different direction of allelic effects between Q4 and the rest of the QTL could be interpreted as evidence that germination time has not been under constant directional selection, because it should prevent the fixation of alleles with antagonistic effects (Orr 1998). However, even under selection, alternative alleles can be driven to fixation by genetic hitchhiking or drift, so the QTL sign test (Orr 1998) generally requires large number of loci to make inferences about selection pressures on a trait (Rieseberg et al. 2002). Therefore, one QTL with opposite

allelic effects relative to other loci does not yet indicate neutrally evolving trait. Especially,

This article is protected by copyright. All rights reserved. 
because the effect sizes of the discovered QTL were fairly large, which usually suggests strong selection (Yeaman \& Whitlock 2011).

Initially, we discovered ten candidate genes within the $95 \%$ confidence intervals of Q3 and Q4. Three of them had promising non-conservative nucleotide substitutions and high $F_{\mathrm{ST}}$ estimates, indicating possible divergent selection (SPY, MAX2 and LHY1). These genes control germination through diverse mechanisms in A. thaliana: SPY has been linked to gibberellic acid mediated germination (Lee et al. 2002), MAX2 is thought to function through growth regulator karrikins and the plant hormone strigolactone (Stanga et al. 2013), while LHY1 is an important mediator of the photoreceptor phytochrome A, and is involved in the light induced germination response (Chen et al. 2014). The confidence intervals of the QTL also contained some strong candidates with neutral $F_{\mathrm{ST}}$ estimates. For example, ABA-INSENSITIVE5 (ABI5), a gene known to be a major regulator of postdormancy germination in A. thaliana (Piskurewicz et al. 2008), localized within Q4. If $A B I 5$, or some other neutrally evolving gene, is actually the causative locus underlying Q4, fixation by genetic drift could explain why that QTL has allelic effects opposite to population averages. Interestingly, none of the discovered QTL colocalized with DOG1, the major dormancy QTL in A. thaliana, which is found in A. lyrata chromosome 8.

\section{Evidence of BDMIs affecting germination}

Besides potentially adaptive variation in germination time, our results revealed evidence of genetic incompatibilities between the subspecies. Hybrid germination proportions were reduced and $F_{2}$ seeds germinated worse than $F_{1}$ seeds - a pattern of inheritance consistent with the Bateson-Dobzhansky-Muller model with partly recessive negatively interacting alleles.

This article is protected by copyright. All rights reserved. 
Hybrid seed germination has primarily been examined between groups that are already reproductively isolated. These encompass species that are fairly diverged (Garner et al. 2016; Oneal et al. 2016), have different ploidy levels (Haig \& Westoby 1991; Ramsey \& Schemske 1998) or mating systems (Rebernig et al. 2015). Also in the genus Arabidopsis, these studies have only been conducted between different species (Burkart-Waco et al. 2012; Muir et al. 2015; Lafon-Placette et al. 2017). However, by studying distinct species, the earliest mechanisms by which reproductive isolation forms are potentially obscured by the accumulation of incompatibility alleles. Within species crosses, in contrast, can yield important insights into these first barriers. In the present study, hybrid germination reduction was modest, but the differences were still distinct and statistically significant. Earlier studies by Leppälä \& Savolainen (2011) and Aalto et al. (2013) found evidence of cytoplasmic male sterility in hybrids between the A. lyrata subspecies, but in the same experiments, seed production did not differ significantly between hybrids and pure-populations. These results, along with the evidence of hybrid vigor (Leinonen et al. 2011), indicate that reproductive isolation between the subspecies is still at its incipient stage, and could break down upon secondary contact. Nevertheless, here we have shown that hybrid seed inviability is one of the first reproductive barriers to form between the A. lyrata subspecies, and therefore a potentially important step in the speciation process.

Patterns of asymmetry between the hybrid reciprocals can be used to make inferences about the causative mechanism. In the first hybrid generation, the progeny with Kar cytoplasm had distinctly reduced germination proportions, whereas the progeny with Ma cytoplasm did not differ from the Ma parental population. In the second generation, however, both reciprocals showed lower seed germination than pure-populations. Several different factors can lead to these types of patterns.

This article is protected by copyright. All rights reserved. 
Cytonuclear interaction are an obvious first choice (Tiffin et al. 2001; Levin 2003; Burton et al. 2013). BDMIs between the Kar cytoplasm and dominant Ma nuclear genes could account for the asymmetry between the $F_{1}$ reciprocals, while additional recessive interactions between Ma cytoplasm and Kar nuclear loci might explain the lack of asymmetry in the $\mathrm{F}_{2}$ generation. However, other mechanism can be just as likely. Several investigations have revealed a role for genomic imprinting in seed endosperm development ( $\mathrm{Li} \&$ Berger 2012) and, consequently, parent-of-origin dependent differences in gene dosage have been implicated in interspecific hybrid seed mortality (Haig \& Westoby 1991; Florez-Rueda et al. 2016; Garner et al. 2016). If imprinted genes are involved in hybrid seed development in our cross, they could lead to asymmetric reproductive isolation in the first hybrid generation, in which the parents are pure-population individuals, but not in the formation of the second generation, as previously paternal and maternal imprinted alleles are mixed in the hybrid parents and the effect should appear symmetrical. However, if the reproductive barrier is only caused by expression imbalance between maternal and paternal alleles of the same loci, the barrier should appear stronger in the $F_{1}$ generation. Therefore, imprinting is not a likely mechanism here, because the $F_{2}$ generation had lower germination proportions than the $F_{1}$ generation, implying that BDMIs with partially recessive interacting alleles are involved in the hybrid inviability. The asymmetry patterns in the $F_{1}$ and $F_{2}$ reciprocals (significant asymmetry in the $F_{1}$ but not in the $F_{2}$ ) could be better explained by incompatibilities between maternal gene products and hybrid genome of the zygote or between maternal diploid component and paternal haploid component of the triploid seed endosperm (Turelli \& Moyle 2007).

This article is protected by copyright. All rights reserved. 


\section{Transmission ratio distortion as an indicator of genetic incompatibilities}

We observed a high degree of transmission ratio distortion in the $F_{2}$ generation of our cross. Many of the distorted markers were clustered in particular areas within the genome and the clustering was more prominent for reciprocals with Ma cytoplasm. Distortion in these clusters was mainly caused by lack of Kar alleles, suggesting the existence of negative interactions between Ma cytoplasmic loci and nuclear genes of Kar origin. There was only minor epistatic TRD between these chromosomes, implying that the loci underlying the TRD act independently.

Several factors associated with genomic divergence can lead to distortion of the genotype ratios: nonrandom segregation of male or female gametes in meiosis (meiotic drive), their differential survival or performance before fertilization, and viability differences between $\mathrm{F}_{2}$ zygotes (Fishman et al. 2001). In theory, any of these factors can cause asymmetric TRD by interacting with the cytoplasmic genomes. Inbreeding depression, another common cause for TRD (Remington \& O’Malley 2000), should not influence these results, because our crossing design avoided mating between relatives and because inbreeding depression is expected to appear symmetrically. Seed mortality, as quantified by the germination experiment, cannot explain all of the TRD in the most heavily distorted areas, where one homozygote was almost completely missing, suggesting that mechanisms acting at earlier stages than seed germination are involved in generating the TRD.

Factors affecting gametes or zygotes are expected to distort the genotypic ratios in a predictable manner (Leppälä et al. 2013). If the heterozygous $\mathrm{F}_{1}$ pollen recipient with Ma cytoplasm only transmits one of its two alleles, but the $F_{1}$ pollen donor with Kar cytoplasm transmits both alleles equally, the resulting MaKar heterozygotes and MaMa homozygotes should to be in 1:1 ratios in the $F_{2}$. Instead, if the distortion is due to viability effects in the

This article is protected by copyright. All rights reserved. 
zygote, with one recessive locus interacting with the Ma cytoplasm, heterozygotes and homozygotes should be in 2:1 ratios. Our results do not fully correlate with either of these scenarios, but in the most heavily distorted areas, the genotype ratios were closer to $1: 1$ ratios (Fig. S8, Supporting information), pointing towards female (only the mothers of the distorted families had Ma cytoplasm) gamete interactions or meiotic drive. However, if the negative interactions are between Ma cytoplasm and only one of the two types of Kar alleles (even though our SNPs were biallelic, each locus in the $\mathrm{F}_{2}$ families contains two Kar and two Ma alleles, which need not be identical), zygote level TRD can mimic gamete inviability by affecting Kar homozygotes and half of the heterozygotes. Therefore, we cannot fully distinguish at what stage the TRD arises, because we cannot follow the maternal and paternal inheritance of alleles.

\section{Conclusions}

Our results show that genetic divergence between the A. lyrata subspecies has diverse effects on seed germination: germination time showed phenotypic differentiation between the populations, while hybrids had lower germination proportions than pure-populations, indicating reproductive isolation. At the genetic level, germination time variation seems to be mainly controlled by two antagonistic QTL, which colocalized with several candidate loci. Three of these genes have evidently been under differential selection and include nonsynonymous substitutions that could account for the phenotypic differences. Possibly driven by the selection, genetic incompatibilities have formed between these subspecies, and the interacting alleles act, at least in part, through uniparentally inherited factors. Hybrid seed mortality was still fairly modest, and the deleterious genotypes could not be deduced from the transmission ratios, which showed patterns of gamete or early zygote inviability. The degree of TRD was, however, high and it was clearly influenced by cytonuclear interactions, suggesting that hybrids have suffered a selective loss of genotypes already before 
germination. These results confirm that the adaptive potential of seed germination is not limited to the dormancy stage and that hybrid seed inviability can be one of the first reproductive barriers to form in Arabidopsis species.

\section{Acknowledgements}

The authors thank Ulla Kemi and David Remington for providing seeds for the crossing experiment, Jaromir Guzinski and Soile Alatalo for help with the sequencing library preparation and David Remington, members of the Plant Genetics Group in Oulu and three anonymous reviewers for their helpful comments on the manuscript. The sequence processing was performed in computer clusters provided by Finnish IT Center for Science (CSC). This work was supported by Biocenter Oulu and Academy of Finland's Research Council for Biosciences and Environment (grant numbers: 136804 and 132611).

\section{References}

Aalto E, Koelewijn HS, Savolainen O (2013) Cytoplasmic Male Sterility Contributes to Hybrid Incompatibility Between Subspecies of Arabidopsis lyrata. G3, 3, 1727-1740.

Alonso-Blanco C, Bentsink L, Hanhart CJ, Blankestijn-de Vries H, Koornneef M (2003) Analysis of natural allelic variation at seed dormancy loci of Arabidopsis thaliana. Genetics, 164, 711-729.

Barnard-Kubow KB, So N, Galloway LF (2016) Cytonuclear incompatibility contributes to the early stages of speciation. Evolution, 70, 2752-2766.

Baskin CC, Baskin JM (1998) Seeds: Ecology, Biogeography, and Evolution of Dormancy and Germination. Academic Press, San Diego, California, USA.

Bates D, Maechler M, Bolker B, Walker S (2016) lme4: Linear mixed-effects models using Eigen and $\mathrm{S} 4$. R package version, 1.

Bateson W (1909) Heredity and variation in modern lights. In: Darwin and Modern Science. (ed Seward AC), pp. 85-101. Cambridge University Press, Cambridge, UK.

Bentsink L, Hanson J, Hanhart CJ et al. (2010) Natural variation for seed dormancy in Arabidopsis is regulated by additive genetic and molecular pathways. Proceedings of the National Academy of Sciences of the United States of America, 107, 4264-4269.

Bentsink L, Jowett J, Hanhart CJ, Koornneef M (2006) Cloning of DOG1, a quantitative trait locus controlling seed dormancy in Arabidopsis. Proceedings of the National Academy of Sciences of the United States of America, 103, 17042-17047.

This article is protected by copyright. All rights reserved. 
Bolger AM, Lohse M, Usadel B (2014) Trimmomatic: A flexible trimmer for Illumina sequence data. Bioinformatics, 30, 2114-2120.

Brink RA, Cooper DC (1947) The endosperm in seed development. The Botanical Review, 13, 423-477.

Broman KW, Wu H, Sen S, Churchill GA (2003) R/qtl: QTL mapping in experimental crosses. Bioinformatics (Oxford, England), 19, 889-890.

Burghardt LTL, Edwards BR, Donohue K (2016) Multiple paths to similar germination behavior in Arabidopsis thaliana. New Phytologist, 209, 1301-1312.

Burkart-waco D, Josefsson C, Dilkes B et al. (2012) Hybrid incompatibility in Arabidopsis is determined by a multiple-locus genetic network. Plant Physiology, 158, 801-812.

Burton RS, Pereira RJ, Barreto FS (2013) Cytonuclear genomic interactions and hybrid breakdown. Annual Review of Ecology, Evolution, and Systematics, 44, 281-302.

Chen F, Li B, Demone J et al. (2014) Photoreceptor partner FHY1 has an independent role in gene modulation and plant development under far-red light. Proceedings of the National Academy of Sciences of the United States of America, 111, 11888-93.

Clauss MJ, Koch MA (2006) Poorly known relatives of Arabidopsis thaliana. Trends in Plant Science, 11, 449-459.

Danecek P, Auton A, Abecasis G et al. (2011) The variant call format and VCFtools. Bioinformatics, 27, 2156-2158.

Debieu M, Tang C, Stich B et al. (2013) Co-Variation between Seed Dormancy, Growth Rate and Flowering Time Changes with Latitude in Arabidopsis thaliana. PLoS ONE, 8.

Dobzhansky T (1936) Studies in hybrid sterility. II. Localization of sterility factors in Drosophila pseudoobscura hybrids. Genetics, 21, 113-135.

Donohue K, Rubio De Casas R, Burghardt L, Kovach K, Willis CG (2010) Germination, postgermination adaptation, and species ecological ranges. Annual Review of Ecology, Evolution, and Systematics, 41, 293-319.

Finch-Savage WE, Leubner-Metzger G (2006) Seed dormancy and the control of germination. New Phytologist, 171, 501-523.

Fishman L, Kelly AJ, Morgan E, Willis JH (2001) A genetic map in the Mimulus guttatus species complex reveals transmission ratio distortion due to heterospecific interactions. Genetics, 159, 1701-1716.

Florez-Rueda AM, Paris M, Schmidt A et al. (2016) Genomic Imprinting in the Endosperm is Systematically Perturbed in Abortive Hybrid Tomato Seeds. Molecular Biology and Evolution, 33, 2935-2946.

Garner AG, Kenney AM, Fishman L, Sweigart AL (2016) Genetic loci with parent-of-origin effects cause hybrid seed lethality in crosses between Mimulus species. New Phytologist, 211, 319-331.

Garrison E, Marth G (2012) Haplotype-based variant detection from short-read sequencing. arXiv preprint arXiv:1207.3907 [q-bio.GN].

Haig D, Westoby M (1991) Genomic Imprinting in Endosperm - its Effect on Seed

This article is protected by copyright. All rights reserved. 
Development in Crosses between Species, and between Different Ploidies of the Same Species, and its Implications for the Evolution of Apomixis. Philosophical Transactions of the Royal Society of London Series B-Biological Sciences, 333, 1-13.

Haley CS, Knott SA (1992) A simple regression method for mapping quantitative trait loci in line crosses using flanking markers. Heredity, 315-324.

Hijmans R, Cameron S, Parra J (2005) Very high resolution interpolated climate surfaces for global land areas. International Journal of Climatology, 25, 1965-1978.

Hu TT, Pattyn P, Bakker EG et al. (2011) The Arabidopsis lyrata genome sequence and the basis of rapid genome size change. Nature Genetics, 43, 476-483.

Jalas J, Suominen J (1994) Atlas Florae Europaea. Distribution of Vascular Plants in Europe. 10: Cruciferae (Sisymbrium to Aubrieta). The Committee for Mapping the Flora of Europe \& Societas Biologica Fennica Vanamo, Helsinki.

Kemi U, Niittyvuopio A, Toivainen T et al. (2013) Role of vernalization and of duplicated FLOWERING LOCUS C in the perennial Arabidopsis lyrata. New Phytologist, 197, 323-335.

Kerdaffrec E, Filiault DL, Korte A et al. (2016) Multiple alleles at a single locus control seed dormancy in Swedish Arabidopsis. eLife, 5, e22502.

Kim E, Donohue K (2013) Local adaptation and plasticity of Erysimum capitatum to altitude: Its implications for responses to climate change. Journal of Ecology, 101, 796-805.

Kronholm I, Picó FX, Alonso-Blanco C, Goudet J, Meaux J De (2012) Genetic basis of adaptation in Arabidopsis thaliana: Local adaptation at the seed dormancy QTL DOG1. Evolution, 66, 2287-2302.

Kuittinen H, De Haan AA, Vogl C et al. (2004) Comparing the linkage maps of the close relatives Arabidopsis lyrata and A. thaliana. Genetics, 168, 1575-1584.

Kärkkäinen K, Løe G, Ågren J (2004) Population structure in Arabidopsis lyrata: Evidence for divergent selection on trichome production. Evolution, 58, 2831-2836.

Lafon-Placette C, Johannessen IM, Hornslien KS et al. (2017) Endosperm-based hybridization barriers explain the pattern of gene flow between Arabidopsis lyrata and Arabidopsis arenosa in Central Europe. Proceedings of the National Academy of Sciences, 201615123.

Lee S, Cheng H, King KE et al. (2002) Gibberellin regulates Arabidopsis seed germination via RGL2, a GAI/RGA-like gene whose expression is up-regulated following imbibition. Genes \& Development, 16, 646-658.

Leinonen PH, Remington DL, Savolainen O (2011) Local adaptation, phenotypic differentiation, and hybrid fitness in diverged natural populations of Arabidopsis lyrata. Evolution, 65, 90-107.

Leinonen PH, Sandring S, Quilot B et al. (2009) Local adaptation in european populations of Arabidopsis lyrata (Brassicaceae). American Journal of Botany, 96, 1129-1137.

Leppälä J, Bechsgaard JS, Schierup MH, Savolainen O (2008) Transmission ratio distortion in Arabidopsis lyrata: effects of population divergence and the S-locus. Heredity, 100, 71-78.

This article is protected by copyright. All rights reserved. 
Leppälä J, Bokma F, Savolainen O (2013) Investigating incipient speciation in Arabidopsis lyrata from patterns of transmission ratio distortion. Genetics, 194, 697-708.

Leppälä J, Savolainen O (2011) Nuclear-cytoplasmic interactions reduce male fertility in hybrids of Arabidopsis lyrata subspecies. Evolution, 65, 2959-2972.

Levin DA (2003) The cytoplasmic factor in plant speciation. Systematic Botany, 28, 5-11.

Li H (2013) Aligning sequence reads, clone sequences and assembly contigs with BWAMEM. arXiv:1303.3997v1 [q-bio.GN].

Li J, Berger F (2012) Endosperm: Food for humankind and fodder for scientific discoveries. New Phytologist, 195, 290-305.

Li H, Durbin R (2009) Fast and accurate short read alignment with Burrows-Wheeler transform. Bioinformatics, 25, 1754-1760.

Lowry DB, Modliszewski JL, Wright KM, Wu CA, Willis JH (2008) The strength and genetic basis of reproductive isolating barriers in flowering plants. Philosophical transactions of the Royal Society of London. Series B, Biological sciences, 363, 30093021.

Manichaikul A, Moon JY, Sen Ś, Yandell BS, Broman KW (2009) A Model Selection Approach for the Identification of Quantitative Trait Loci in Experimental Crosses, Allowing Epistasis. Genetics, 181, 1077-1086.

Montesinos-Navarro A, Picó FX, Tonsor SJ (2012) Clinal Variation In Seed Traits Influencing Life Cycle Timing In Arabidopsis thaliana. Evolution, 66, 3417-3431.

Muir G, Ruiz-Duarte P, Hohmann N et al. (2015) Exogenous selection rather than cytonuclear incompatibilities shapes asymmetrical fitness of reciprocal Arabidopsis hybrids. Ecology and Evolution, 5, 1734-1745.

Muller HJ (1939) Reversibility in evolution considered from the standpoint of genetics. Biological reviews of the Cambridge Philosophical Society, 14, 261-280.

Muller M-H, Leppälä J, Savolainen O (2008) Genome-wide effects of postglacial colonization in Arabidopsis lyrata. Heredity, 100, 47-58.

Oneal E, Willis JH, Franks RG (2016) Disruption of endosperm development is a major cause of hybrid seed inviability between Mimulus guttatus and Mimulus nudatus. New Phytologist, 210, 1107-1120.

Van Ooijen JW (2011) Multipoint maximum likelihood mapping in a full-sib family of an outbreeding species. Genetics research, 93, 343-349.

Orr HA (1998) Testing Natural Selection vs. Genetic Drift in Phenotypic Evolution Using Quantitative Trait Locus Data. Genetics, 149, 2099-2104.

Penfield S, Springthorpe V (2012) Understanding chilling responses in Arabidopsis seeds and their contribution to life history. Philosophical Transactions of the Royal Society of London. Series B, Biological sciences, 367, 291-297.

Peterson BK, Weber JN, Kay EH, Fisher HS, Hoekstra HE (2012) Double digest RADseq: An inexpensive method for de novo SNP discovery and genotyping in model and nonmodel species. PLoS ONE, 7.

This article is protected by copyright. All rights reserved. 
Piskurewicz U, Jikumaru Y, Kinoshita N et al. (2008) The gibberellic acid signaling repressor RGL2 inhibits Arabidopsis seed germination by stimulating abscisic acid synthesis and ABI5 activity. The Plant Cell, 20, 2729-2745.

Postma FM, Ågren J (2015) Maternal environment affects the genetic basis of seed dormancy in Arabidopsis thaliana. Molecular Ecology, 24, 785-797.

Postma FM, Ågren J (2016) Early life stages contribute strongly to local adaptation in Arabidopsis thaliana. Proceedings of the National Academy of Sciences, 113, 75907595.

Pyhäjärvi T, Aalto E, Savolainen O (2012) Time scales of divergence and speciation among natural populations and subspecies of Arabidopsis lyrata (Brassicaceae). American Journal of Botany, 99, 1314-1322.

Quilot-Turion B, Leppälä J, Leinonen PH et al. (2013) Genetic changes in flowering and morphology in response to adaptation to a high-latitude environment in Arabidopsis lyrata. Annals of Botany, 111, 957-968.

R Core Team (2015) R: A language and environment for statistical computing. $R$ Foundation for Statistical Computing, Vienna, Austria.

Ramsey J, Schemske DW (1998) Pathways, mechanisms, and rates of polyploid formation in flowering plants. Annual Review of Ecology and Systematics, 29, 467-501.

Rebernig CA, Lafon-Placette C, Hatorangan MR, Slotte T, Köhler C (2015) Non-reciprocal Interspecies Hybridization Barriers in the Capsella Genus Are Established in the Endosperm. PLoS Genetics, 11.

Remington DL, Figueroa J, Rane M (2015) Timing of shoot development transitions affects degree of perenniality in Arabidopsis lyrata (Brassicaceae). BMC plant biology, 15, 1.

Remington DL, O'Malley DM (2000) Whole-genome characterization of embryonic stage inbreeding depression in a selfed loblolly pine family. Genetics, 155, 337-348.

Rieseberg LH, Widmer A, Arntz AM, Burke JM (2002) Directional selection is the primary cause of phenotypic diversification. Proceedings of the National Academy of Sciences of the United States of America, 99, 12242-5.

Rieseberg LH, Widmer A, Arntz AM, Burke JM (2003) The genetic architecture necessary for transgressive segregation is common in both natural and domesticated populations. Philosophical Transactions of the Royal Society B-Biological Sciences, 358, 1141-1147.

Riihimäki M, Savolainen O (2004) Environmental and genetic effects on flowering differences between northern and southern populations of Arabidopsis lyrata (Brassicaceae). American Journal of Botany, 91, 1036-1045.

Stanga JP, Smith SM, Briggs WR, Nelson DC (2013) SUPPRESSOR OF MORE AXILLARY GROWTH2 1 controls seed germination and seedling development in Arabidopsis. Plant Physiology, 163, 318-30.

Tiffin P, Olson MS, Moyle LC (2001) Asymmetrical crossing barriers in angiosperms. Proceedings of the Royal Society B: Biological Sciences, 268, 861-867.

Trabucco A, Zomer R. (2009) Global Aridity Index (Global-Aridity) and Global Potential Evapo-Transpiration (Global-PET) Geospatial Database. CGIAR Consortium for Spatial

This article is protected by copyright. All rights reserved. 
Information, Published online: http://www.csi.cgiar.org.

Turelli M, Moyle LC (2007) Asymmetric postmating isolation: Darwin's corollary to Haldane's rule. Genetics, 176, 1059-1088.

Vergeer P, Kunin WE (2013) Adaptation at range margins: Common garden trials and the performance of Arabidopsis lyrata across its northwestern European range. New Phytologist, 197, 989-1001.

Wagmann K, Hautekeete NC, Piquot Y et al. (2012) Seed dormancy distribution: explanatory ecological factors. Annals of Botany, 110, 1205-1219.

Weir BS, Cockerham CC (1984) Estimating $F$-statistics for the analysis of population structure. Evolution, 38, 1358-1370.

Yeaman S, Whitlock MC (2011) The genetic architecture of adaptation under migrationselection balance. Evolution, 65, 1897-1911.

\section{Data Accessibility}

-DNA sequences: NCBI SRA: PRJNA357693 ( $F_{2}$ progeny), SAMN06141193 -

SAMN06141198 (Ma individuals) and SAMN06159138 - SAMN06159141 (Kar individuals).

-Phenotype data, mapping files and R scripts: Dryad doi:10.5061/dryad.q713h

\section{Author Contributions}

T.H., P.H.L. and O.S. designed the study. T.H. conducted the experiments, analyzed the data and wrote the manuscript with H.K. and O.S. T.M.M. provided data. H.K. and O.S. supervised the study. All authors commented on the manuscript.

This article is protected by copyright. All rights reserved. 




Figure 1. Germination phenotypes for parental populations Karhumäki, Russia (Kar) and Mayodan, North Carolina (Ma) and $F_{1}$ and $F_{2}$ hybrid reciprocals. A: Germination times (thick line: median, box: upper and lower quartiles, whiskers: \pm 1.5 interquartile range, dots: outliers). B: Average germination proportions and \pm 1 standard errors. Colors indicate cytoplasmic origin $($ red $=$ Kar, blue $=\mathrm{Ma}$ ). Statistical significances of the pair-wise tests are shown above brackets: $* P<0.05, * * P<0.001, * * * P<0.0001$.

This article is protected by copyright. All rights reserved. 


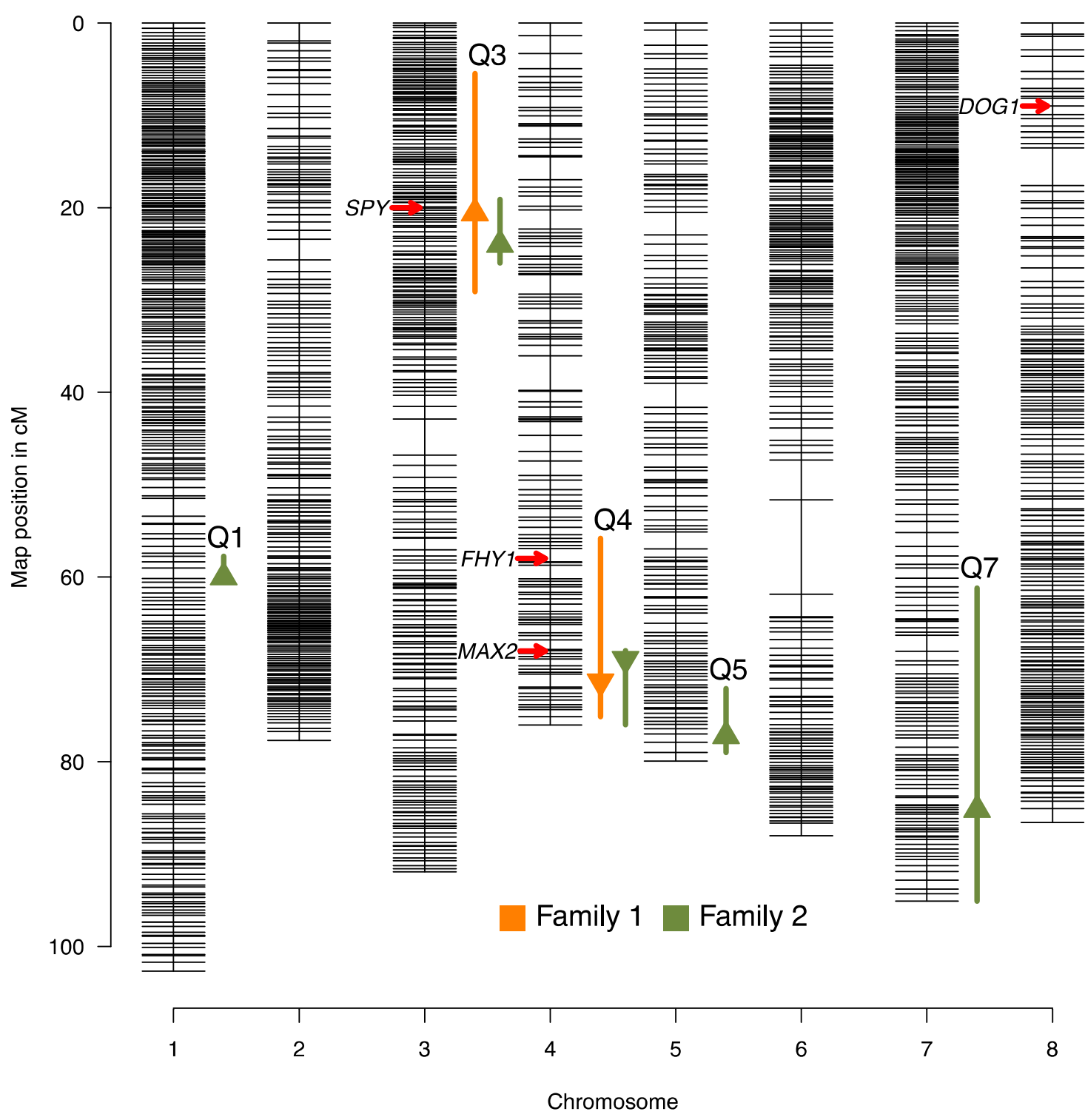

Figure 2. Genome-wide significant $(P<0.05)$ QTL for germination time. Line shows the 95\% confidence interval for QTL locations and arrow marks the most likely point estimate. Arrow direction shows the effect of the Kar homozygote (upwards: faster germination, downwards: slower germination). Q5 and Q7 showed cytonuclear interactions with significant effect in Kar background and the effect direction is shown for individuals with that cytoplasm. Approximate positions of the candidate genes and DOG1 are also shown.

This article is protected by copyright. All rights reserved. 

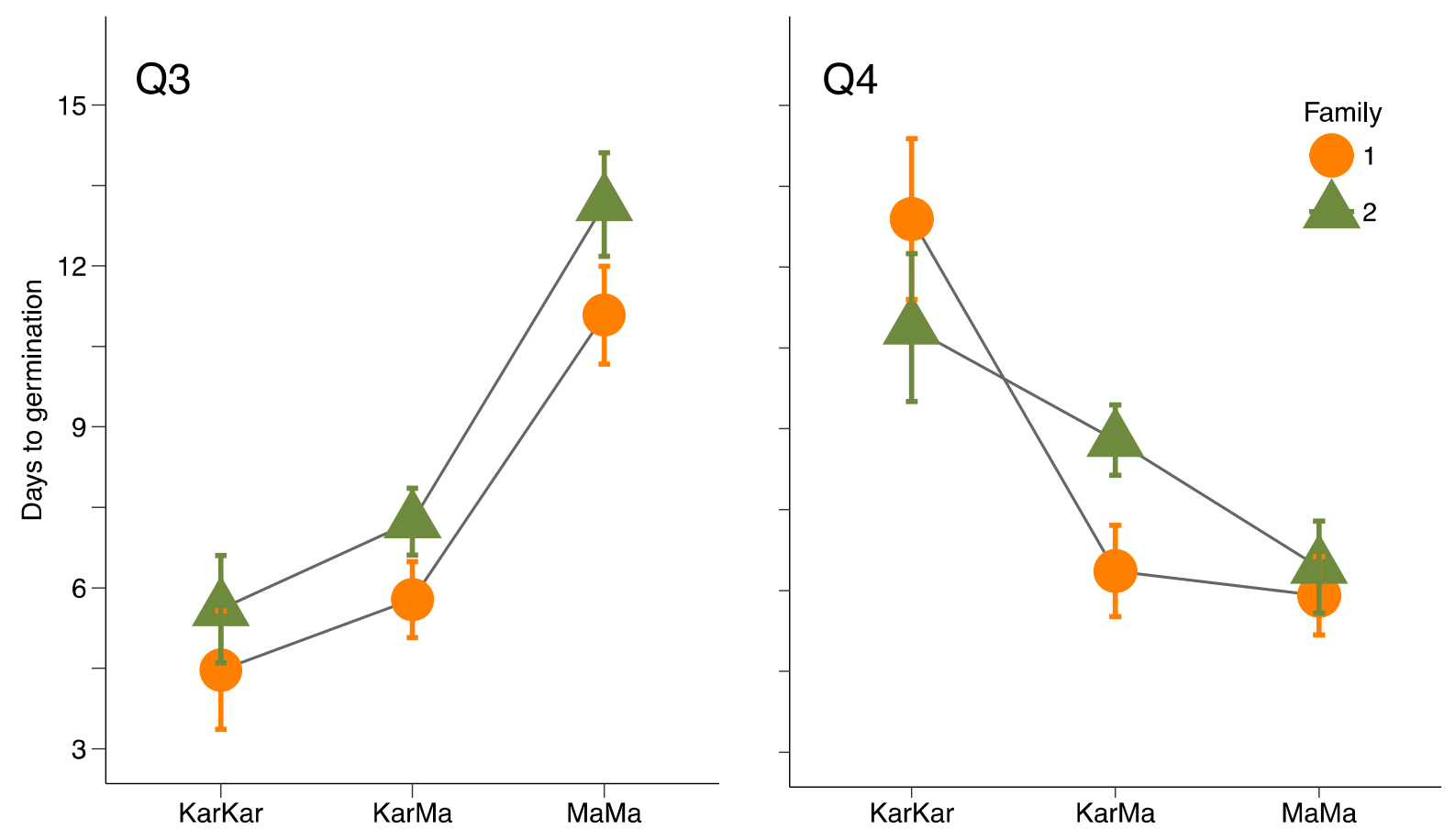

Figure 3. Mean germination times and \pm 1 standard errors for genotypes in the two large effect QTL found in both families.

This article is protected by copyright. All rights reserved. 

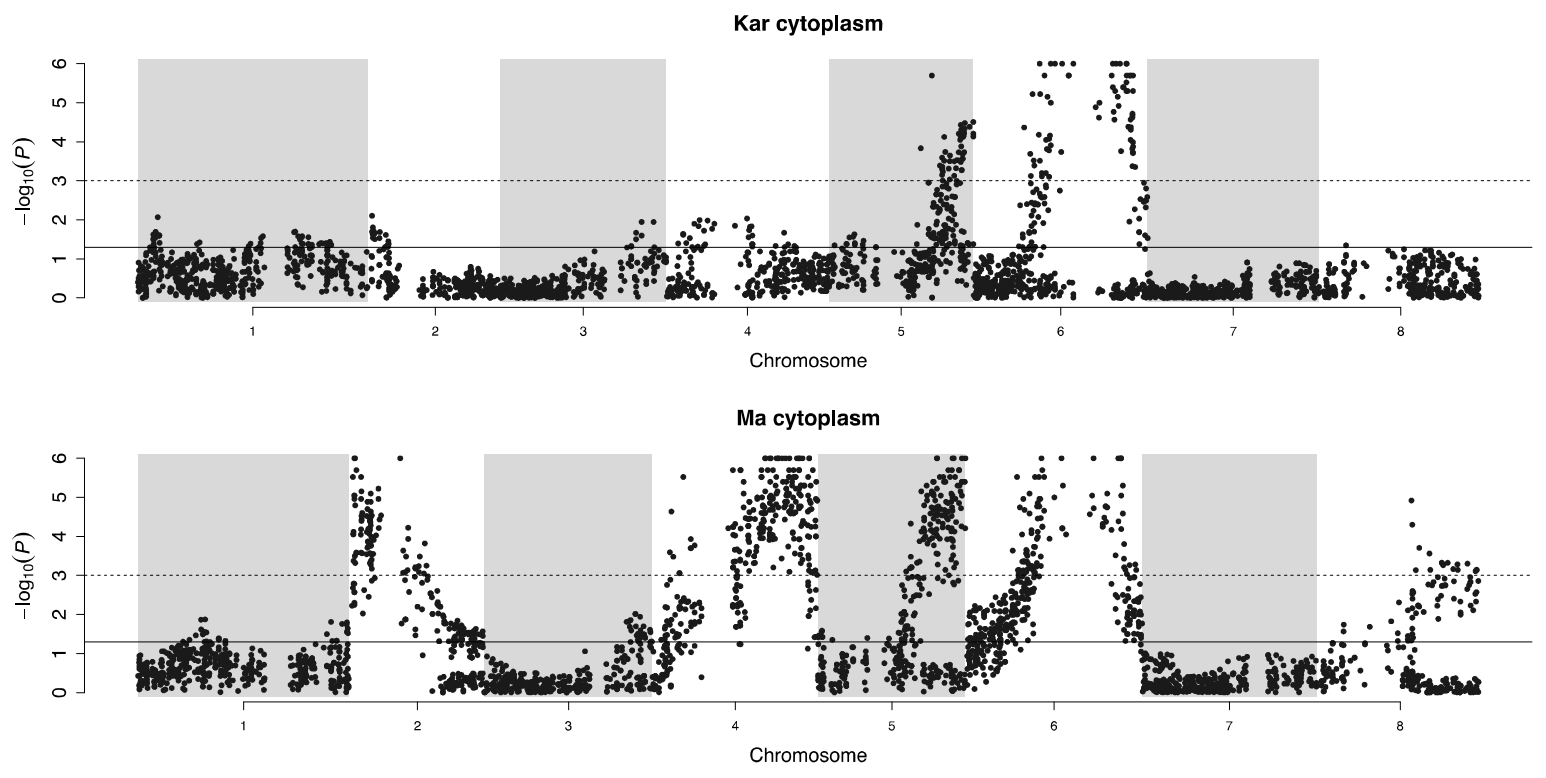

Figure 4. Genome-wide patterns of transmission ratio distortion in $F_{2}$ reciprocals with Kar and Ma cytoplasms for the two families combined. $Y$-axis shows the $-\log _{10}$ transformed $P$ values for SNP-by-SNP $\chi^{2}$ tests with d.f. $=2$ for 3087 and 3117 biallelic SNPs, respectively. To focus on the TRD clusters, suspected false positives were filtered from the figure by scanning the chromosomes in 30 SNP sliding windows, calculating mean and SD for the $P$ values, and removing any SNP that deviated more than one SD from the mean (for unfiltered $P$-values see Fig. S2, Supporting information). Solid line marks the significance threshold at $\alpha=0.05$ level and dashed line at $\alpha=0.001$ level. The physical position along the chromosomes is shown in the $x$-axis.

This article is protected by copyright. All rights reserved. 


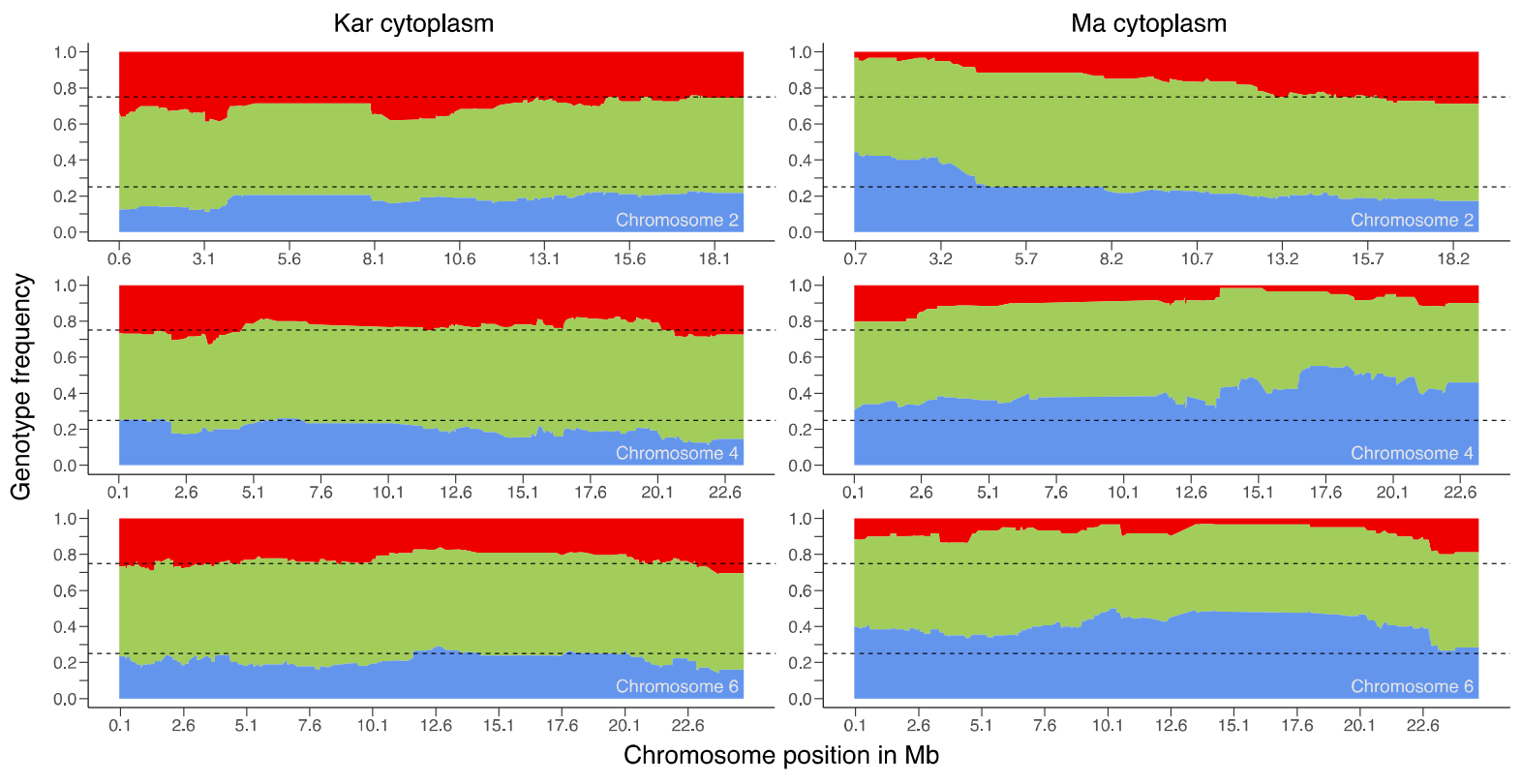

Figure 5. Genotype frequencies of the heterozygotes (green) and Mayodan (blue) and Karhumäki (red) homozygotes in chromosomes 2, 4 and 6 showing TRD clusters in the two families. Both reciprocals of one family $(n=172)$ are shown. Dashed lines mark the expected frequencies.

This article is protected by copyright. All rights reserved. 
Table 1. QTL for germination time. For each significant $(P<0.05)$ QTL, family from which it was discovered from, chromosome, position in $\mathrm{cM}$ and percentage of variance explained (PVE) are shown. Effect is calculated as the difference between mean germination times of the two homozygotes. A positive value means faster germination for Kar homozygote and a negative value faster germination for Ma homozygote.

\begin{tabular}{|c|c|c|c|c|c|}
\hline QTL & Family & Chromosome & $\begin{array}{c}\text { Position } \\
\text { (95\% Bayes) }\end{array}$ & PVE & Effect \\
\hline Q1 & 2 & 1 & $\begin{array}{c}60.0 \\
(57.7-60.6)\end{array}$ & 15.6 & 2.10 \\
\hline Q3 & 1 & 3 & $\begin{array}{c}20.6 \\
(5.5-29.1)\end{array}$ & 17.4 & 6.62 \\
\hline Q3 & 2 & 3 & $\begin{array}{c}24.0 \\
(19.1-26.0)\end{array}$ & 28.0 & 7.54 \\
\hline Q4 & 1 & 4 & $\begin{array}{c}71.3 \\
(55.8-75.1)\end{array}$ & 18.2 & -6.98 \\
\hline Q4 & 2 & 4 & $\begin{array}{c}68.9 \\
(67.9-76.0)\end{array}$ & 10.1 & -4.44 \\
\hline $\mathbf{Q 5}^{\mathbf{a}}$ & 2 & 5 & $\begin{array}{c}79.2 \\
(72.1-81.2)\end{array}$ & $11.4^{\mathrm{b}}$ & $2.45^{\mathrm{b}}$ \\
\hline $\mathbf{Q 7}^{\mathbf{a}}$ & 2 & 7 & $\begin{array}{c}85.2 \\
(61.2-95.1)\end{array}$ & $17.0^{\mathrm{b}}$ & $7.19^{b}$ \\
\hline
\end{tabular}

${ }^{a}$ Cytonuclear interaction, with significant effect in Kar background.

${ }^{\mathrm{b}}$ Among individuals with Kar cytoplasm.

This article is protected by copyright. All rights reserved. 